\title{
COBÁ Y TULUM: ADAPTACIÓN AL MEDIO AMBIENTE Y CONTROL DEL MEDIO SOCIAL*
}

\author{
Antonio Benavides Castillo \\ Centro Regional del Sureste, INAH
}

Los objetivos principales de este ensayo son dos: 1) informar sobre las investigaciones recientes realizadas en Cobá y en Tulum con respecto al patrón de asentamiento; 2) derivar de la información an. terior algunas reflexiones acerca del urbanismo entre los mayas antiguos.

El recorrido de ambas zonas arqueológicas del estado de Quintana Roo nos ha proporcionado información más detallada de diversos elementos fisiográficos que fueron considerados por los mayas prehispá. nicos en la distribución de sus asentamientos. En esta forma empezamos a definir con mayor precisión no sólo los diversos sectores dedicados a la habitación sino también aquellos dedicados a la explotación del medio geográfico en ambos sitios.

\section{Cobá}

Empecemos por la ciudad con el mayor sistema de caminos construidos de Mesoamérica. Las primeras noticias arqueológicas de Cobá proceden prácticamente de finales de la década 1920-1930, cuando la Institución Carnegie de Washington realizó varias expediciones. Como observación preliminar se habló entonces de que la zona cubría un rectángulo aproximado de $3.5 \mathrm{~km}$ (E-W) por $2 \mathrm{~km}$ (N-S) y que había sitios periféricos vinculados al espacio anterior por medio de caminos o sacbés, pero sin considerarlos como partes integrantes de un solo sitio (Thompson et al., 1932: 18).

* Ponencia presentada en el simposio "Interdisciplinary approaches to Maya studies", del XLIII Congreso Internacional de Americanistas. Vancouver, Canadá, agosto $10-17,1979$.

Estudios de Cultura Maya. Vol. XIII, 1981

Instituto de Investigaciones Filológicas/

Centro de Estudios Mayas, UNAM

http://www.iifilologicas.unam.mx/estculmaya/ 
Casi medio siglo después, investigadores del INAH realizamos el levantamiento topográfico de la red de caminos (Fig. 1), lo cual permitió obtener una idea más clara de la extensión y forma del asentamiento. En esa ocasión se calculó una extensión mínima de 70 km² (Benavides, 1976b: 160) a la vez que se obtuvo la secuencia cronológica del sitio mediante el estudio de la cerámica (Robles, en prensa).

Paralelamente a los trabajos del INAH en Cobá, la National Geographic Society financió varios recorridos del sitio, cuya extensión estimaron en $61.5 \mathrm{~km}^{2}$ (Folan et al., 1977), para luego concentrar sus esfuerzos en un sector norteño de Cobá (Fletcher, 1978; Kintz, 1978).

Hoy día el INAH prosigue reuniendo material de campo y de gabinete para estudiar el patrón de asentamiento de la zona. Se planteó así, en lo que al campo respecta, localizar y describir todo vestigio arqueológico hallado a lo largo de una muestra formada por cuatro líneas o brechas (N, S, E y W) que parten del núcleo de Cobá. Las brechas norte y sur tienen, cada una, $5 \mathrm{~km}$ de longitud por $200 \mathrm{~m}$ de ancho. Las brechas este y oeste tienen la misma anchura pero su longitud sólo es de $2.5 \mathrm{~km}$.

Con la información producto de las brechas se esperaba conocer la extensión total del sitio y obtener una muestra representativa de la diversidad arqueológcia. Sin embargo, los resultados recientes nos indican que Cobá cubre una superficie mayor a $l o s 70 \mathrm{~km}^{2}$ antes mencionados. Con base en esto se ha pensado ampliar la muestra durante las próximas temporadas.

$\mathrm{Al}$ recorrer cada eje se registra todo elemento arqueológico visible a la vez que se hacen observaciones sobre el entorno. En esta forma se obtienen anotaciones y planos de las plataformas, de los cimientos de casas habitación, de las bardas, de los edificios techados con bóveda, de los metates, de los cimientos circulares, etc. De manera similar se anota la presencia de las diversas fuentes permanentes o temporales de agua, de las sascaberas o canteras, e incluso se observa el tipo de suelo asociado a los parajes recorridos (Garduño, 1977).

Por otra parte, conscientes de la importancia que pudieron haber tenido los suelos de la zona en la distribución del asentamiento, hemos entrevistado a personas de la localidad a la vez que complementamos la información, en lo posible, mediante los datos proporcionados por fotos aéreas de la zona.

En planta, el asentamiento de Cobá es atravesado horizontalmente por grandes fajas o bandas de suelos de varios kilómetros de extensión (Leonardo Falcón K., comunicación personal, 1978) (Fig. 2). Básicamente hallamos dos tipos de terreno: ek luum (tierra negra, también llamada box luum) y chac luum (tierra roja), aunque también 


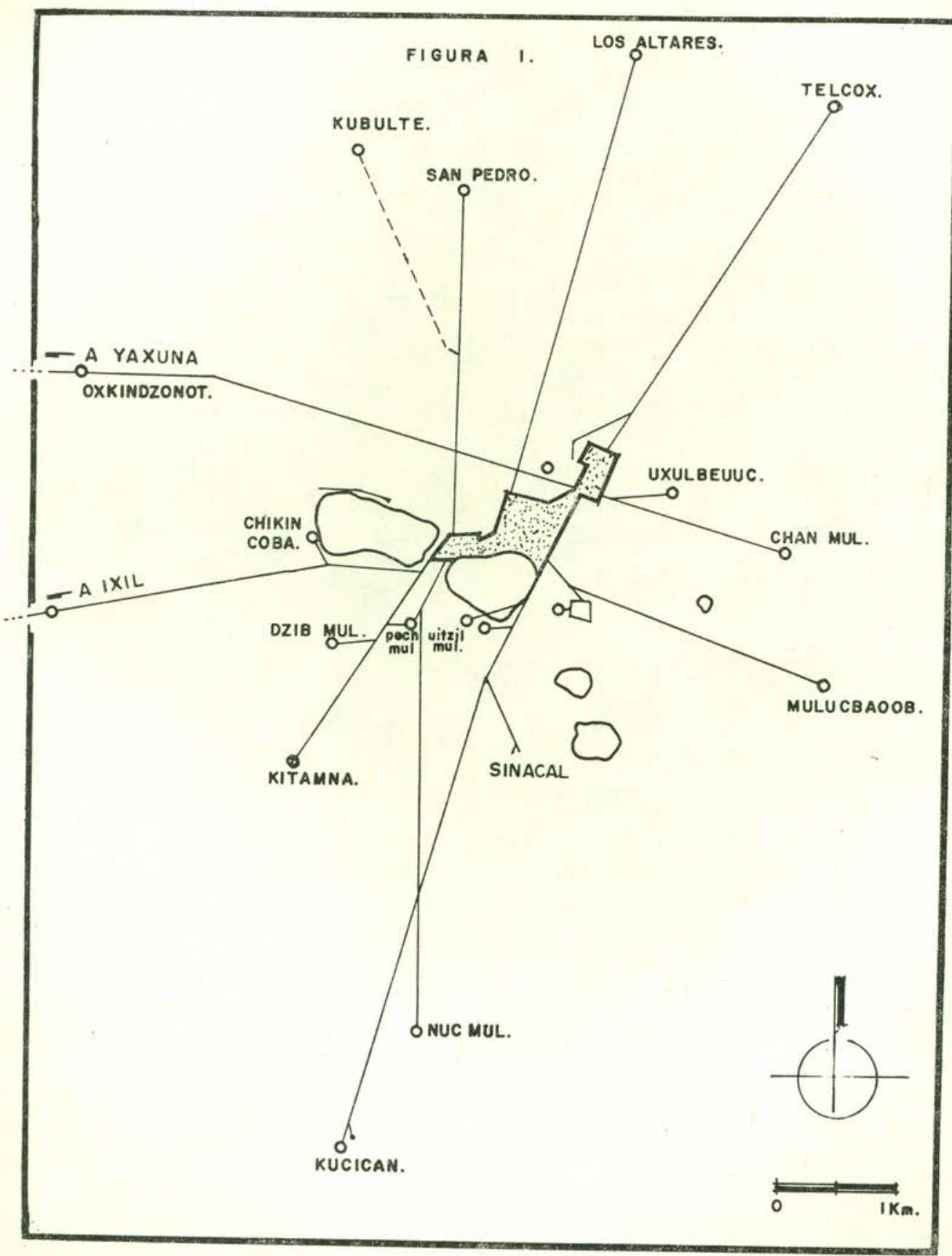


FIOURA 2
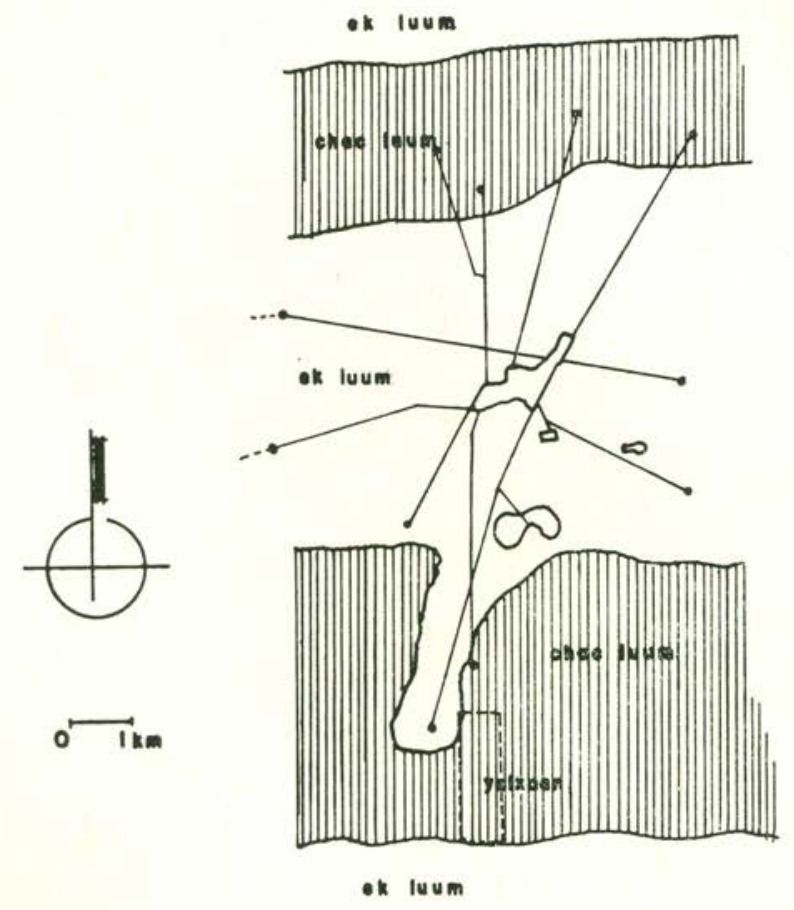

- lo lum

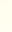

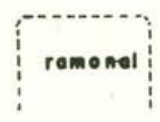


se detectaron sectores de sac luum (tierra blanca). Este último tipo de suelo está distribuido irregularmente, como manchas, y por lo general existe en los akalchés o bajos inundables en época de lluvias.

La variedad ek luum es la más fértil y precisamente sobre una ancha faja de ella se hallan los cinco lagos de la zona y la mayor parte del asentamiento monumental cobaéense. Las excepciones son los sitios terminales del norte (Kubulté, San Pedro, Los Altares y Telcox), que se hallan sobre chac luum; Kitamná (al sureste) sobre sac luum y Nuc Mul (al sur) sobre chac luum.

Los límites norte y sur de la gran banda central de ek luum están marcados por el inicio de fajas de chac luum tras las cuales reapare. cen terrenos de ek luum. El sac luum es el menos abundante y se concentra al oriente y al sur de los lagos.

\section{Tulum}

En cuanto a la antigua Zamá, los inicios de las exploraciones arqueológicas también se remontan al decenio 1920-30. Lothrop (1924) publica un plano bastante completo del sector amurallado de Tulum, a la vez que reporta abundante información sobre el sitio y otros va. rios de la costa oriental de Quintana Roo.

Otra importante aportación es aquélla de Sanders (1960), bási. camente en lo que respecta a la secuencia cronológica. Sin embargo, su información sobre la distribución del asentamiento debe tomarse con precaución, pues asegura la inexistencia de unidades habitacionales entre Tancah y Tulum cuando en realidad sí las hay. También dice que el asentamiento prehispánico de Tancah sólo cubre una superficie aproximada de $0.5 \mathrm{~km}^{2}$, pero nuevamente sin haber visto buen número de plataformas, bardas y cimientos de casas habitación que existen más allá de la retícula por él utilizada y considerada como límite de los vestigios arqueológicos.

Los recorridos de superficie recientemente efectuados al sur y al norte del famoso sector amurallado de Tulum (Velázquez, 1976; Barrera, 1977) han revelado que existen unidades habitacionales delimitadas por muros sencillos o dobles de unos $0.50 \mathrm{mts}$ de altura promedio.

Los muros parten de las esquinas noroeste y suroeste de la muralla, corren a lo largo del límite del manglar (localizado al poniente) y se adaptan a la elevación natural del terreno. De dichos muros se desprenden bardas hacia el oriente, quedando así muchos predios o solares prehispánicos entre la playa y el pantano de manglar. Por el sur, un 
muro doble o andador (es decir una especie de largo pasillo formado por bardas a ambos lados) se extiende por espacio de unos $3 \mathrm{~km}$. siempre atendiendo a las irregularidades de las orillas del manglar y de la playa. En cuanto al sector norte, los vestigios arqueológicos de Tulum y de Tancah (distantes unos $3 \mathrm{~km}$ entre sí) llegan a confundirse de tal manera (Tomás Gallareta, comunicación personal, 1978) que en realidad podemos hablar de un solo asentamiento.

En esta forma tenemos una comunidad longilínea de unos $8 \mathrm{~km}$ de largo por $200 \mathrm{~m}$ de ancho, es decir con una superficie aproximada de poco más de $1.5 \mathrm{~km}^{2}$. Esta superficie debe considerarse como mínima pues de hecho desconocemos la verdadera superficie ocupada por los sectores habitacionales al poniente y al norte de Tancah, así como aquéllos al sur de Tulum.

Un pasaje del itinerario de Juan de Grijalva permite complementar la información arqueológica. Al empezar a relatar el recorrido por el litoral oriental de Quintana Roo en 1518 el documento menciona: ". . . un pueblo o aldea tan grande que la ciudad de Sevilla no podría parecer mayor ni mejor... por la costa andaban muchos indios... haciéndose señas de que nos acercásemos; pero el capitán no quiso" (Díaz, 1958: 23). Si los españoles no desembarcaron y hablan de un gran asentamiento, con mucha gente y "muchas casas de paja" todo ello debió verse a lo largo de la costa.

\section{Usos del espacio en Cobá}

Uno de los criterios principales que utilizamos para hablar de la distribución del asentamiento precolombino es la concentración o la dispersión de los restos arqueológicos en el espacio. Encontramos así un continuum de elementos cuyas densidades y características varían según nos hallemos en uno $u$ otro punto del sitio. También hemos tratado de considerar la cantidad y la calidad de las construcciones observadas. Esto significa tomar en cuenta tanto el número de edificios hallado, como sus dimensiones y características, entre las que se advierte la simetría de su disposición, espacios construidos y no cons. truidos, nivelaciones artificiales del terreno, etc.

Procedimos así a analizar el primer cuerpo de información con el que contamos: el corazón de Cobá y sus sistemas de caminos. El estudio de los 43 sacbeob y de los 24 sitios terminales hasta hoy registrados nos permitió diferenciar varios usos del espacio ocupado por la zona de Cobá. En términos generales encontramos tres subdivisiones espaciales a las que denominamos subzonas: nuclear, perinuclear y exte- 
rior. La identificación de estas subzonas se amolda en gran medida a los elementos arquitectónicos monumentales (grupos centrales. Tsacbeob zonales y grupos periféricos) de Cobá. Por esta razón será necesario afinar dicha subdivisión con base en la información que nos proporcionan multitud de bardas, plataformas y cimientos de casas en sectores pertenecientes a Cobá pero alejados de los elementos monumentales mencionados.

Veamos ahora las características principales de las subzonas hasta ahora observadas en Cobá (Fig. 3).

\section{Subzona Nuclear}

El corazón de Cobá cubre una superficie aproximada de $2 \mathrm{~km}^{2}$ y se halla básicamente sobre terrenos de ek luum. La fuerza de trabajo concentrada en esta subzona creó una enorme nivelación artificial que sirve de base a tres grandes grupos arquitectónicos o acrópolis: Cobá, Nohoch Mul y Chumuc Mul. Esta magna obra adopta en planta la silueta de una $L$ invertida y cubre una superficie aproximada de $0.5 \mathrm{~km}^{2}$. En el extremo sureste de esta Gran Nivelación comienza el sacbé 9 , que puede considerarse como una prolongación de dicha nivelación para unir al Grupo Macanxoc con los anteriores.

En la subzona nuclear se originan los caminos regionales ( 1 y 16 ) que llevan a Yaxuná e Ixil, así como los demás que proporcionan cohesión interna a toda la zona. Los restos de construcciones hoy visibles en los cuatro grupos centrales son básicamente monumentales. Entre ellos sobresalen la Gran Plataforma, el Nohoch Mul y La Iglesia. Además existen numerosos edificios con múltiples crujías dispuestas en uno o dos niveles. También hay espacios abiertos monumentales como las plazas principales de los grupos Cobá y Nohoch Mul. Las estelas y los altares del sitio también están concentradas en esta subzona.

Los materiales cerámicos y arquitectónicos presentes en la subzona nuclear nos hablan de todas las etapas de la vida prehispánica del sitio. La mayor parte de la construcción total de esta subzona corres. ponde al periodo Clásico y ocasionalmente se hallan unidades habitacionales (que en lo sucesivo abreviaremos como UH) del Postclásico sobre las plazas monumentales. 


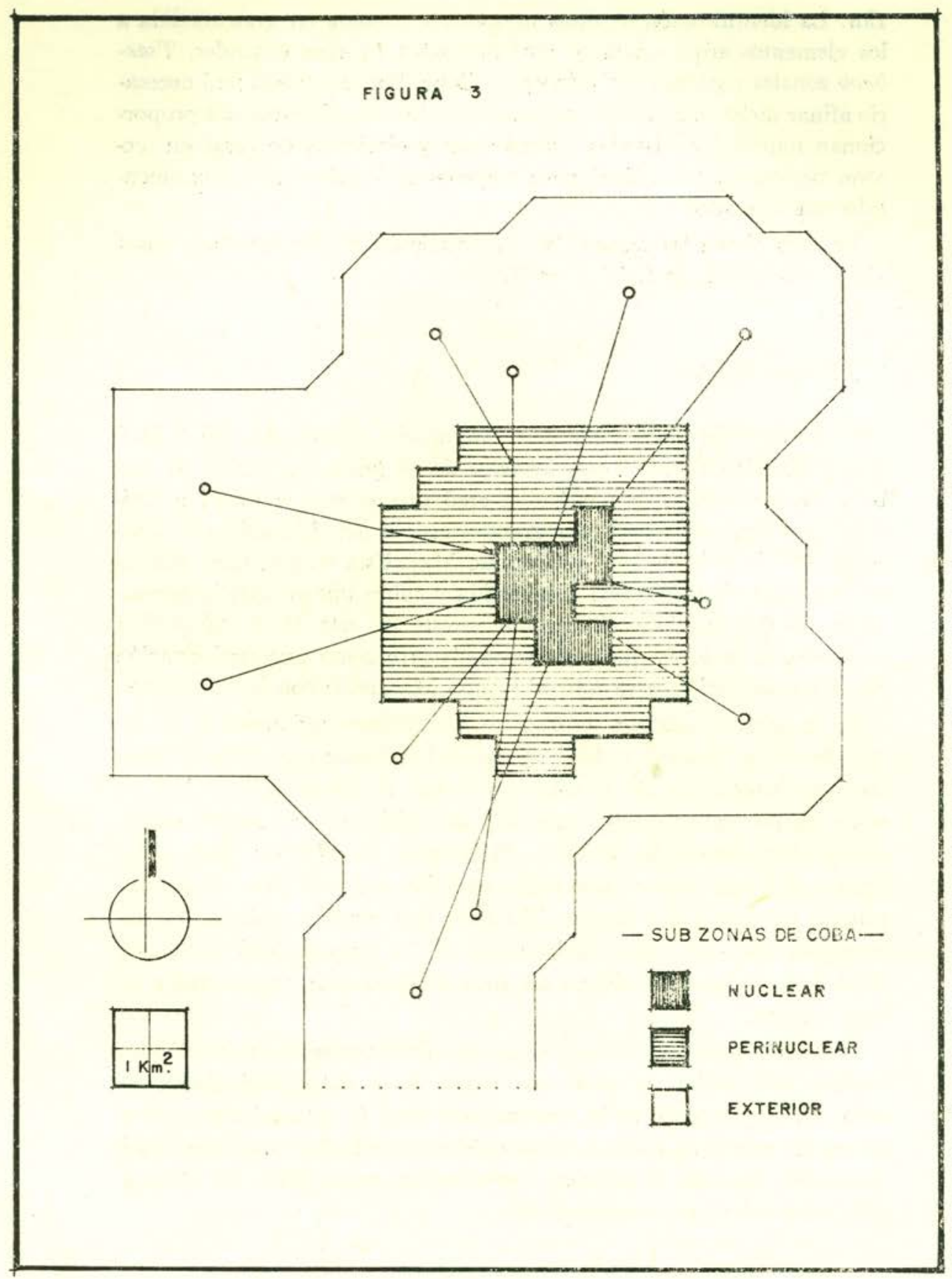

Estudios de Cultura Maya. Vol. XIII, 1981

Instituto de Investigaciones Filológicas/

Centro de Estudios Mayas, UNAM

http://www.iifilologicas.unam.mx/estculmaya/ 


\section{Subzona Perinuclear}

En esta subzona tenemos construcciones de dimensiones mucho menores que en el núcleo de Cobá y se hallan también sobre la faja central de ek luum. Asimismo, encontramos conjuntos de edificios techados con bóveda, pero no tantos como antes. Algunos ejemplos son los sitios terminales Dzib Mul (Benavides y Robles, 1976), Uxulbeuuc y Chikín Cobá. Otros conjuntos de edificios más modestos incluyen El Cuartel (Benavides, 1976a: 52-62) y las estructuras excavadas en el predio asignado a un hotel (Terrones y Trejo, 1977). Empero, hay muchísimos vestigios más de construcciones hechas con materiales perecederos y cimientos de piedra que cubren prácticamente toda la subzona.

Tentativamente hemos calculado $13 \mathrm{~km}^{2}$ para la subzona perinuclear, superficie que, a grosso modo, rodea al núcleo de Cobá, aunque de manera irregular por tener que conformarse a las características fisiográficas (lagos, akalchés, etc.) y sociales (Gran Nivelación, grupos monumentales centrales, etc.) del centro de la antigua urbe.

En la parte interior de la subzona perinuclear parece existir una especie de cinturón o espacio delimitador caracterizado por una baja densidad de restos arqueológicos (Garduño, 1977, planos). Aparentemente las UH del Clásico se construyeron a unos $500 \mathrm{~m}$ alejadas de la subzona nuclear. A esa distancia sí se observa una fuerte concentra. ción de UH (generalmente rodeadas por bardas) que contiene construcciones de calidad diversa, la mayoría orientadas a los puntos cardinales.

La explicación de este fenómeno puede hallarse al considerarlo somo un reflejo de la diferenciación socio-económica existente en la sociedad maya del Clásico. En el posible cinturón mencionado también se encuentran UH aunque en menor densidad y con características distintas: la orientación de los edificios es diferente y las bardas que encierran conjuntos son menos regulares.

Si la observación con respecto a la temporalidad de las UH antes mencionadas es correcta, las halladas cerca de la subzona nuclear deberán corresponder al periodo Postclásico. La presencia de estas últimas en ese lugar y para esa época podría explicarse en función de haber desaparecido ya un rigor de ubicación domiciliaria antes vigente. Futuras excavaciones podrán proporcionar más luz al respecto a la vez que nos permitirán fecha las UH con certeza. 


\section{Subzona Exterior}

A juzgar por la información hasta ahora obtenida esta subzona es la más extensa. En ella se asentaba la mayoría de los habitantes de la ciudad. Cubre una superficie promedio de $60 \mathrm{~km}^{2}$ y los vestigios arqueológicos se hallan más dispersos, no presentan tanta uniformidad en cuanto a orientación y, en términos generales, son de dimensiones menores a los hallados en las subzonas anteriores.

La subzona exterior de Cobá es la más difícil de definir debido a su gran extensión y a su propiedad de envolver o rodear por completo a las subzonas antes vistas. Sus contornos probablemente adoptan una forma amiboidea en los límites del asentamiento global debido a diversos factores: 1) la curva irregular formada por el contorno exterior de la subzona perinuclear; 2) la distinta localización y extensión de los sitios terminales a los cuales también envuelve y 3 ) las posibilidades buenas o malas de acceso al agua y a la tierra cultivable que se hallaren en las afueras de la ciudad.

Un detalle que amerita mención, es la mayor o menor concentración de evidencias arqueológicas en la subzona exterior en proporción directa a la mayor o menor distancia con respecto a los sitios terminales, mismos que también se localizan en esta subzona. En otras palabras, alrededor de los grupos satélites monumentales se notan concentraciones de UH (Jaime Garduño, comunicación personal, 1977) como indicando el poder de cohesión de esos puntos que además cuentan con un sacbé procedente del corazón del sitio.

Casi todos los grupos satélites de Cobá reflejan, a otra escala y con características propias, el núcleo de la vieja urbe. Los elementos monumentales básicos de la subzona nuclear se hallan reproducidos en los grupos terminales: una amplia plaza rodeada por grandes construcciones entre las que sobresale un basamento piramidal, misma que es el origen y fin de una larga y ancha calzada de piedra.

La mayor parte de los grupos terminales se halla también sobre una nivelación artificial del terreno, la que a su vez se encuentra aprovechando una elevación natural.

Suponemos que la mayor parte del asentamiento de la subzona exterior corresponde a los periodos Clásico Medio y Tardío (600-750-1100 d. C.) pues sus vestigios cubren los sectores formados por el curso de los saćeob, tan típicos de la zona y fechados para esa época (Robles, en prensa).

En esta forma, en la subzona exterior parecen existir UH estrecha. mente ligadas a los grupos terminales, así como la subzona perinu 
clear parece corresponder al dominio directo de la subzona nuclear. En este sentido la subzona exterior correspondería al área de influencia de cada sitio terminal, sin que por ello dejaran de pertenecer al gran centro controlador. Es ilustrativo recordar los barrios coloniales o algunas colonias modernas, con puntos focales que repiten las características de la plaza principal o centro aunque no por ello dejan de constituir un todo integrado.

Por lo que respecta a los recorridos efectuados mediante las brechas, hoy sabemos que la densidad del asentamiento disminuye por el norte al llegar a los cinco kilómetros. En el eje E.W las evidencias arqueológicas prosiguen más allá de los dos y medio km y por el sur las UH continúan después de los cinco kilómetros. Incluso se observó que por ese lado falta recorrer un buen trecho para encontrar los límites del asentamiento (Jaime Garduño, comunicación personal, 1978).

$\mathrm{Al}$ comentar los hechos con gente de la zona se nos informó que al sur de Cobá existen dos nivelaciones artificiales o embutidos de considerable extensión. El primero es el de Yalxaan. Tiene unos $2 \mathrm{~km}$ de largo por $700 \mathrm{mts}$. de ancho y se halla justo al sureste de Kucican, el sitio terminal más alejado del corazón de Cobá por este lado (Leonardo Falcón, comunicación personal, 1978). El segundo embutido, el de Ramonal, comienza a unos $5 \mathrm{kms}$ al sureste de Kucican, tiene unos $6 \mathrm{kms}$ de largo por $2 \mathrm{kms}$ de ancho y su eje mayor también está orientado de norte a sur (José Isabel Cocom, comunicación personal, 1978).

Las rancherías modernas pertenecientes al Ejido Ruinas de Cobá se hallan asentadas precisamente sobre dichos embutidos o cerca de ellos, pues ahí cuentan con buenas tierras para la agricultura. Un detalle que refuerza la opinión de los informantes es el significado de los topónimos dados a los embutidos. Yalxaan significa "abundancia de guano", es decir de la palma conocida con ese nombre (Reinhardtia sp y Sabal mayarum). Ramonal se refiere a la alta frecuencia del árbol ramón (Brosimum alicastrum). Como todos sabemos, el campesino moderno prefiere cultivar los terrenos que se hallan en las zonas arqueológicas, pues en ellos encuentra una mayor fertilidad, una mayor duración de las propiedades del suelo y consecuentemente mejores cosechas.

Ante esta información la próxima temporada deberemos verificar la extensión espacial de Yalxaan y Ramonal a fin de replantear el estudio del asentamiento de Cobá por el lado sur. 
Usos del espacio en Tancah-Tulum

En cuanto al asentamiento de Tancah-Tulum podemos diferenciar cuatro subzonas, dos monumentales y dos habitacionales (Fig. 4). En general, se aprovechó una elevación natural del terreno que corre paralela al litoral. Las subzonas monumentales parecen localizarse precisamente en donde existe un relieve con mayor amplitud.

1. Subzona Tancah monumental. Aquí se concentran edificios de mampostería y plataformas que requieren de una fuerte inversión de fuerza de trabajo. En total se cubre una superficie de 20 has, es decir, la quinta parte de un $\mathrm{km}^{2}$. En esta subzona también encontramos las evidencias cerámicas más tempranas del asentamiento, empezando en el Preclásico Medio y finalizando en la época colonial (Miller, 1977).

II. Subzona Tancah-Tulum habitacional. Se extiende al sur de la subzona anterior y al norte del sector amurallado de Tulum, entre el mar y el manglar. Básicamente está integrada por bardas dobles y sencillas que delimitan solares en los que encontramos plataformas, cimientos de casas habitación, altarcitos (Tomás Gallareta, comuni. cación personal, 1978) e incluso edificios de mampostería como los llamados extra-muros a medio kilómetro al norte de la muralla de Tulum. La superficie aproximada de esta subzona es de 60 has. o sea poco más de $0.5 \mathrm{~km}^{2}$.

III. Subzona Tulum monumental. Este sector es el que se conoce internacionalmente, el famoso rectángulo turístico delimitado por una gruesa muralla. Ocupa 6 has en las que nuevamente tenemos una concentración de edificios de mampostería y de grandes plataformas que soportaron habitaciones de materiales perecederos. En esta subzona las construcciones presentan una distribución bastante ordenada. Es factible distinguir dos ejes longitudinales sobre los que queda alineada la mayoría de los edificios a la vez que los demás presentan sus muros orientados con la misma regularidad.

$I V$. Subzona Tulum habitacional. Al sur de la muralla de Tulum se extiende otra vez una franja de terreno elevado entre la playa y el pantano de manglar. Dicha franja presenta restos arqueológicos de unidades habitacionales como las descritas en la subzona TancahTulum habitacional. La densidad de elementos arqueológicos (bardas, plataformas, cimientos, metates, etc.) aumenta con respecto a la cercanía al sector amurallado. La extensión mínima de esta subzona es de 80 has. 


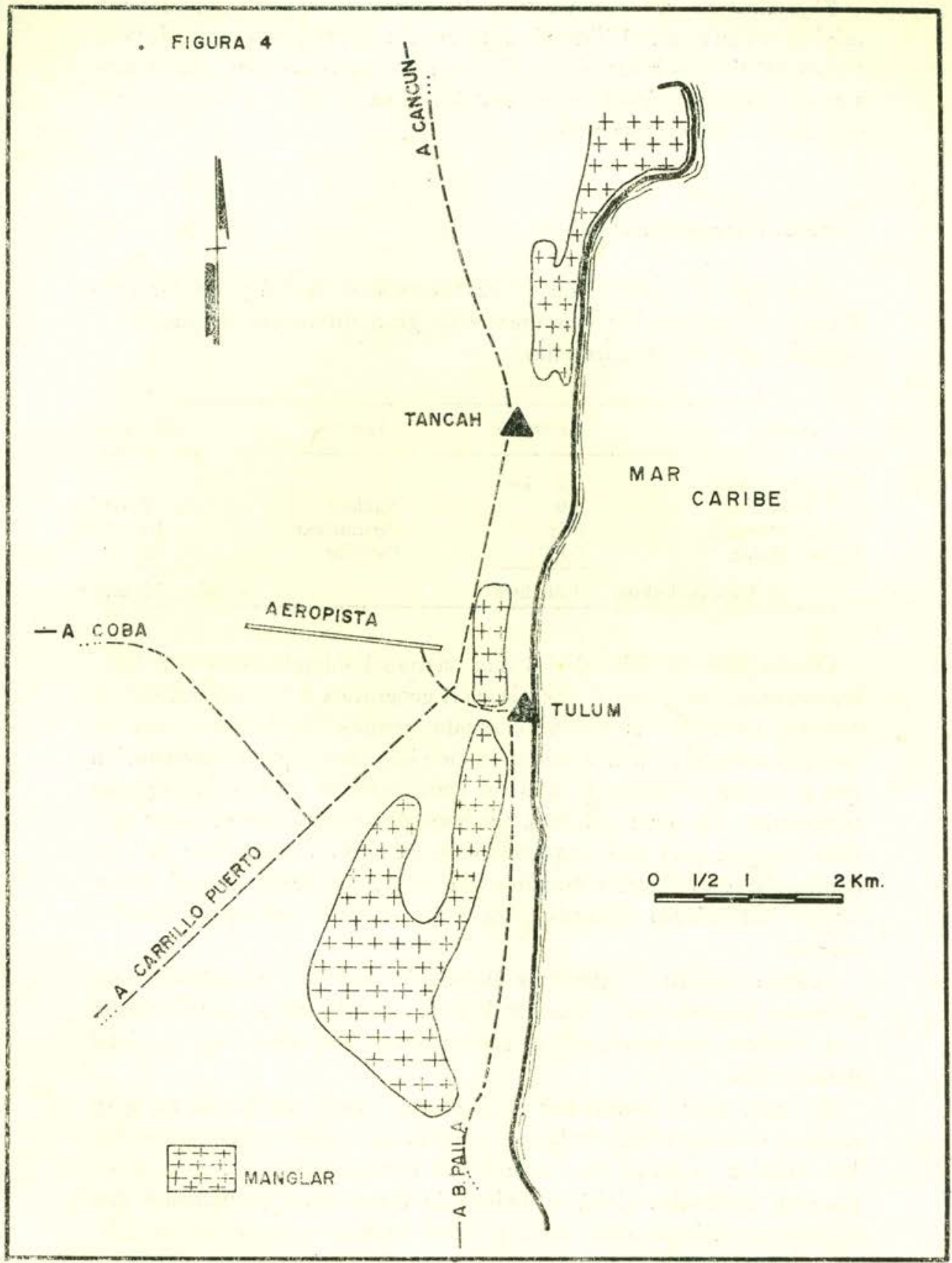

Estudios de Cultura Maya. Vol. XIII, 1981 Instituto de Investigaciones Filológicas/ Centro de Estudios Mayas, UNAM 
El análisis de la cerámica da la impresión de que el asentamiento original ocurrió en el Tancah monumental para luego expanderse a través del tiempo hacia el sur. En esta forma lo que hoy apreciamos son las concentraciones monumentales (una al norte y otra al sur) postclásicas del mismo sitio.

\section{Ciudad: desigualdad social}

Al comparar la extensión de las subzonas de Cobá y de TancahTulum advertimos inmediatamente la gran diferencia en superficie ocupada que existe entre ellas:

\begin{tabular}{llllr}
\hline \multicolumn{1}{c}{ Subzona } & Extensión & Subzona & Extensión \\
\hline Tancah Monum. & $0.2 \quad \mathrm{~km}^{2}$ & & \\
T-T Habit. & 0.6 & Nuclear & $2 \mathrm{~km}^{2}$ \\
Tulum Monum. & 0.06 & & Perinuclear & 13 \\
Tulum Habit. & 0.8 & & Exterior & 60 \\
\cline { 2 - 2 } Tancan-Tulum & $1.66 \mathrm{~km}^{2}$ & & Cobá & $75 \mathrm{~km}^{2}$ \\
\hline
\end{tabular}

Obviamente los sitios de los que estamos hablando presentan fuertes discrepancias entre sí (localización geográfica y por ende acceso a recursos naturales; superficie ocupada; temporalidad; etc.), mas lo que nos interesa destacar ahora es precisamente aquella información que sí puede contrastarse, aun sin considerar las diferencias espaciotemporales. En otras palabras, tenemos dos sitios diferentes por muchas razones, pero que nos hablan de un mismo fenómeno: la concentración de esfuerzos humanos en puntos concretos con el conse. cuente crecimiento desigual para otros sectores del mismo asentamiento.

¿Cómo explicar en términos sociales lo anterior? En primer lugar debemos comenzar por desechar la falsa premisa de estar trabajando con "centros ceremoniales" o asentamientos con una baja densidad demográfica.

Si hasta ahora carecíamos de evidencias para pensar en un gran número de habitantes asociado a un sitio arqueológico era por no ha. ber visto la multitud de vestigios no monumentales asociada a las grandes pirámides. Cobá y Tulum lo demuestran plenamente con grandes extensiones, cada una a su nivel, de terreno cubiertos por UH. Estos datos vienen a sumarse a los ya conocidos en otras localidades del área maya: 


\begin{tabular}{lccl}
\hline \multicolumn{1}{c}{ Sitio } & $K m^{2}$ & Población & \multicolumn{1}{c}{ Referencia } \\
\hline Mayapán & 4.2 & 11500 & Pollock et al., 1962: 211 \\
Tikal & 163 & 49000 & Haviland, 1969: 430 \\
Dzibilchaltún & 19 & 42000 & Kurjack, 1974: 94 \\
Chunchucmil & 20 & 12000 & Vicek et al., 1978: 217 \\
\hline
\end{tabular}

Debemos agregar que para la ciudad de Cobá del Clásico Tardío existe un cálculo de población preliminar (de 42400 a 70009 habitantes) basado en una muestra de $3.5 \mathrm{~km}^{2}$ (Kintz, 1978: 261). Sin embargo, esta estimación incluye severas reducciones debidas al uso no habitacional de edificios, a la supuesta no contemporaneidad y con base en una superficie $\left(61.5 \mathrm{kms}^{2}\right)$ que en realidad resulta menor a la hallada por nosotros $\left(75 \mathrm{kms}^{2}\right)$, misma que seguramente aumentará al finalizar los recorridos complementarios de las brechas (N, S, E y W) y el reconocimiento de los embutidos. A nuestro juicio es necesario contar con información más completa sobre la extensión y temporalidad de las UH de Cobá para poder hablar de demografía en un momento dado.

En cuanto a Tancah-Tulum el trabajo hasta ahora realizado no permite obtener más que cálculos de población muy tentativos como el de 9000 pensado por Sanders (1960: 210, 216), si acaso existieran UH. Como hemos visto, resulta que sí las hay, ahora falta conocerlas y estudiarlas.

Sin embargo, el urbanismo no debe definirse por los kilómetros cuadrados ocupados o por el número de individuos, sino por las relaciones sociales institucionalizadas que estos últimos guardan entre sí. Lo que nos lleva a diferenciar las ciudades de una manera rápida es su no similitud con los asentamientos rurales, es decir con aquéllos en los que no existe ni una fuerte concentración de bienes y servicios ni una marcada heterogeneidad social.

En mayor o menor medida todas las ciudades son el resultado de un proceso que comenzó con el conocimiento y control del medio para luego fortalecerse y crecer mediante el control de las relaciones habidas entre los hombres. Todos los asentamientos urbanos, pasados y presentes, son el fruto de la concentración de un producto social excedente.

Despojémonos también del prejuicio de solamente ver ciudades donde hay retículas. La clave del fenómeno urbano no estriba en la forma sino en el contenido, en cómo se produce la concentración de artefactos y construcciones que el arqueólogo encuentra.

La manufactura de edificios grandes y suntuosos requirió de la par- 
ticipación de buen número de personas, no sólo de aquellas ocupadas en las labores específicas de la albañilería, de la extracción y acarreo de materiales o de los acabados exteriores y finales. También se necesitó del concurso de quienes organizaron y dirigieron los trabajos $\mathrm{y}$, por supuesto, de quienes satisfacieron las necesidades alimenticias de los grupos de individuos antes mencionados. Recuérdese también que toda obra, una vez concluida, requería de mantenimiento periódico, lo cual implica nuevamente una organización afín.

La congregación de habitaciones que cubren una gama de mayor a menor trabajo invertido en varias decenas de hectáreas o de kilómetros cuadrados no puede verse como un hecho sin trascendencias sociales profundas. Las evidencias arqueológicas de Cobá y de Tulum (orientación astronómica; distribución geométrica de edificios monumentales; caminos locales y zonales dando cohesión al asentamiento; UH delimitadas por albarradas; etc.) revelan un modo propio de planificación, una manera específica de cumplir funciones urbanas de diversa índole (económicas, religiosas, políticas, estéticas, etc.).

Lo anteriormente planteado significa forzosamente la existencia de fuertes contrastes o diferencias entre los habitantes de un sitio. La contemporaneidad de viviendas dentro y fuera de un sector amurallado o bien la mayor o menor cantidad de material utilizado en la construcción de uno u otro de los grupos satélites de Cobá es bien explicable si pensamos en función de individuos con un mayor o menor acceso a los bienes y a los servicios producidos por la sociedad. En otras palabras, detrás de una casa grande y otra chica, o de una acrópolis y una unidad habitacional, debemos ver que existía determinado tipo de relaciones entre los hombres cuya desigualdad es evidente.

Los logros arquitectónicos, matemáticos y artísticos (entre otros) de los antiguos mayas nos parecen desproporcionados al considerar el nivel tecnológico o el grado de desarrollo de las fuerzas productivas alcanzado por la sociedad. Pero debemos pensar que la carencia de animales de carga y tiro, así como la falta de innovaciones técnicas más allá de los instrumentos de piedra pulida fueron ampliamente compensadas mediante una organización social clasista, fuertemente jerarquizada, cuyo excelente funcionamiento tuvo como base primordial la excesiva explotación del trabajo humano y de los recursos naturales accesibles para el máximo beneficio de una minoría. Esta fuerte contradicción se resolvería después básicamente mediante un violento rechazo de la mayoría trabajadora al orden impuesto. 


\section{BIBLIOGRAFIA}

Barrera R., Alfredo

1977 "Exploraciones arqueológicas en Tulum, Quintana Roo", en Boletín de la Escuela de Ciencias Antropológicas de la Universidad de Yucatán (ECAUDY), No. 24: 23-63. Zamná, Mérida.

Benavides C., Antonio

1976a "Cobá, Quintana Roo, Reporte de actividades", en Cobá: un sitio maya en Quintana Roo. Cuadernos de los Centros No. 26: 1-104. Dirección de Centros Regionales. INAH, México.

1976b El sistema prehispánico de comunicaciones terrestres en la región de Cobá, Quintana Roo, y sus implicaciones sociales. Tesis. Escuela Nacional de Antropología e Historia. México.

Benavides C., Antonio y Robles C., Fernando

1976 "Cobá: sus sacbeob y Dzib Mul", en Boletín del INAH. Êp. II, No. 15: 55-58. INAH. México.

Díaz, JuAN

1958 Itinerario de Juan de Grijalva, 1518. C. R. Menéndez ed. Mérida.

Fletcher, Laraine A.

1978 Sociocultural implications of the linear features at Cobá, Quintana Roo, Mexico. Tesis. State University of New York. Stony Brook, New York.

Folan, William J.; Stuart, George E.; Fletcher, L. A. y Kintz, Ellen R. 1977 "El proyecto arqueológico-cartográfico de Cobá. Informes interinos (sic) Nos. 1, 2 y 3", en Boletín de la ECAUDY, No. 22-23: 14-81. Zamná. Mérida.

Garduño A., Jaime

1977 "Estudio del patrón de asentamiento de Cobá. Planteamientos para discusión". Mecanuscrito y planos en poder del Centro Regional del Sureste del INAH. Mérida.

Haviland, William A.

1969 "A new population estimate for Tikal, Guatemala", en American Antiquity, 34: 429-433.

Kintz, Ellen Rose

1978 The social organization of a Classic Maya City: Cobá, Quintana Roo, Mexico. Tesis. State University of New York. Stony Brook, New York.

KurJack, Edward B.

1974 Prehistoric lowland Maya community and social organization. Middle American Research Institute. Pub. 38. Tulane University. New Orleans.

Lothrop, SAMUel K.

1924 Tulum: an archaeological study of the East Coast of Yucatan. Carnegie Institution of Washington. Pub. 335. Washington.

Miller, Arthur G.

1977 "The Maya and the sea: trade and cult at Tancah and Tulum, Quintana Roo, Mexico", en The sea in the pre-columbian world; Elizabeth P. Benson ed. 96-138. Dumbarton Oaks Research Library \& Collections. Trustees for Harvard University. Washington. 
Pollock, Harry E. D.; Roys, Ralph L.;

Proskouriakoff, T. y Smith, A. Ledyard

1962 Mayapan, Yucatan, Mexico. Carnegie Institution of Washington. Pub. 619. Washington. (Smith: "Residential and associated

Robles C., FERnando structures at Mayapan"; 165-319.)

En prensa "Secuencia cerámica preliminar de la región de Cobá, Quintana Roo" ponencia presentada en la Mesa Redonda de la Sociedad Mexicana de Antropología celebrada en Guanajuato, Gto., 1977. México.

Sanders, William T.

1960 Prehistoric ceramics and settlement patterns in Quintana Roo, Mexico. Carnegie Institution of Washington. Pub. 606, Contrib 60. Washington.

Thompson, J. Eric; Pollock, H. E. D. y Charlot, Jean

1932 A preliminary study of the ruins of Cobá, Quintana Roo, Mexico. Carnegie Institution of Washington, Pub. 424. Washington.

Terrones G., Enrique y Trejo A., Elia del Carmen

1977 "Salvamento arqueológico en Cobá. Reporte de actividades 1976-1977'. Mecanuscrito en poder del Centro Regional del Sureste del INAH. Mérida.

Velázquez V., Ricardo

1976 "Informe de las exploraciones arqueológicas y trabajos de mantenimiento en la zona de Tulum, Q. R., 1975". en Cuadernos de los Centros, No. 27: 19-83. Dirección de Centros Regionales. INAH. México.

Vlgek, David T.; Garza de González, Sylvia y Kurjack, Edward B.

1978 "Contemporary farming and ancient Maya settlements: some disconcerting evidence", en Prehispanic Maya Agriculture: 211223. Ed. por Harrison P. D. y Turner II, B. L. University of New Mexico Press. Albuquerque. 